\title{
Association between peripheral vascular disease and other macrovascular and microvascular complications in diabetic patients
}

Gonzalo Allo Miguel, Elena García Fernández, Milagros Sierra Bracamonte, Guillermo Martínez Díaz-Guerra, Federico Hawkins

Endocrinology Service, 12 de Octubre University Hospital.

\section{Introduction}

Diabetes mellitus is a condition on the increase, carrying a high risk of macro and microvascular disease. Previous studies suggest a link between microvascular and macrovascular complications in diabetes. However, just a few studies have investigated whether the presence of peripheral vascular disease (PVD) is associated with other macrovascular and microvascular complications in diabetes patients.

Aim

The aim of our study is to evaluate whether the presence of peripheral vascular disease is associated with other macrovascular and microvascular complications in diabetic patients.

\section{Patients and methods}

98 diabetic patients (68 with type 2 and 20 with type 1 diabetes) were included. PVD was confirmed by arteriography. Other macrovascular complications were collected, including: Adverse cardiovascular events (myocardial infarction, angina, etc) and stroke. Microvascular complications were, also, collected: Retinopathy, nephropathy and peripheral neuropathy. Statistical analysis: Chi-square test was used to compare the existence of macro and microvascular complications between the groups with and without PVD.

\section{Results}

48 male and 50 female with mean age $(52.58 \pm 20.70)$ and mean $\mathrm{HbA} 1 \mathrm{c}(11.21 \pm 2.33 \%)$ were included. 14 patients $(15.73 \%)$ were diagnosed of PVD. The existence of PVD was significantly correlated with the existence of more macrovascular complications $(p<0.001)$ and, al least, one microvascular complication $(p<0.005)$. Also, significant differences between the groups of patients with or without PVD were found in the rate of neuropathy $(78.57 \%$ and $23.61 \%$ respectively: $p<0.001)$; cardiovascular events $(50.00 \%$ and $9.59 \%$ respectively: $p=0.002)$ and in the rate of strokes $(28.57 \%$ and $6.67 \%$ respectively; $p=0.01)$. There were no significant differences in the rate of retinopathy and nephropathy between both groups.

\begin{tabular}{|l|c|c|c|}
\hline & PVD - (n=75) & PVD + (n= 14) & p-value \\
\hline $\begin{array}{l}\text { Peripheral } \\
\text { Neuropathy }\end{array}$ & $17(23.61 \%)$ & $11(78.57 \%)$ & $<0.001$ \\
\hline $\begin{array}{l}\text { Cardiovascular } \\
\text { Events }\end{array}$ & $7(9.59 \%)$ & $7(50.00 \%)$ & 0.002 \\
\hline \begin{tabular}{l} 
Strokes \\
\hline
\end{tabular} & $5(6.67 \%)$ & $4(28.57 \%)$ & 0.01 \\
\hline
\end{tabular}

Table 1. Patients with peripheral vascular disease have significant higher rates of peripheral neuropathy, cardiovascular events and strokes.

\section{Conclusions}

Our results show significant correlation between the existence of PVD and the existence of more macrovascular complications and one or more microvascular complications. We have found that diabetic patients with PVD present significant higher rates of neuropathy, cardiovascular events and strokes. Despite the limitations of the study, our results suggest that diabetic patients with PVD require proper micro and macrovascular complications screening. 